\title{
The Study on the Necessity of Learning Medical Physics in Local Medical University in the Policy of the University Transformation
}

\author{
Benchao Zhu ${ }^{1, \text { a }}$, Weiwei Jiang ${ }^{2, b}$ and Fan Zhang ${ }^{2, c}$ \\ ${ }^{1}$ Hubei University of Medicine, Mathematics and Physics Department, Shiyan 442000, China \\ ${ }^{2}$ Hubei Dongfeng Automobile Technician College, Shiyan 442000, China

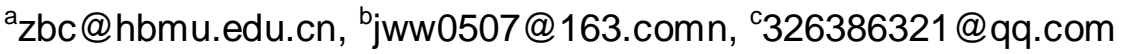

Keywords: The university transformation; Medical physics; Necessity; Education reformation

\begin{abstract}
Under policy of the ministry of education of the transformation of higher schools into skilled occupation education, this paper focus on the public basic course- "medical physics" in the ordinary local medicine colleges. The actual situations are first presented in this paper, then, one can find there are two different interpretations about the transformation policy of higher school in the local medicine colleges. Based on the different understandings of transformation policy will give two different conclusions, the paper respectively analyzes the learning necessity of the "medical physics" course. Particularly, there is always a misunderstanding of learning necessity and existence necessity in all the local medical colleges. Finally, by the analysis and description of the situation of "medical physics" course under the transformation policy, we wish to appeal the university staff should pay more attention to the scientific of reformation of the public basic course.
\end{abstract}

\section{高校转型形势下地方医学院校开设《医学物理学》 课程必要性的研究}

\author{
朱本超 ${ }^{1, \mathrm{a}}$, 蒋薇薇 ${ }^{2, \mathrm{~b}}$, 张钒 ${ }^{2, \mathrm{c}}$ \\ 1. 湖北医药学院, 数理教研室, 中国 湖北 十堰 442000 \\ 2. 湖北东风汽车技师学院, 中国 湖北十堰 442000

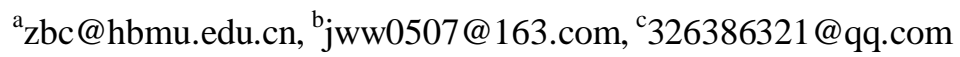

摘要：在教育部出台高等院校转型为应用型、技能型教育政策前提下，本文以地方医学院校 的公共基础课程一 《医学物理学》作为研究对象。从地方医学院校开设的《医学物理学》课 程现状出发, 地方医学院校对高校转型政策的两种不同解读, 分别论述地方医学院校是否还 有开设《医学物理学》课程必要性。在解读政策具有差异的背景下, 给出了开设课程必要性 和不必要性的两种截然不同的结论, 并对该结论的科学性给出了分析。值得注意的是, 笔者 重点分析了当下高校普遍存在的一种认识误区, 即将开设某课程的 “必要性” 理解为该课程 的 “存在性”。最后, 本文旨在通过地方医学院校中的《医学物理学》课程在高校转型政策下 的处境，呼吁高校从业人员重视公共基础课程改革方式的科学性。

关键词: 高校转型; 医学物理学; 必要性; 教学改革

\section{1. 引言}

2014 年 2 月 26 日, 国务院常务会议部署加快构建以就业为导向的现代职业教育体系, 引导 一批普通本科高校向应用技术型高校转型，并将此作为即将印发的《国务院关于加快发展现 代职业教育的决定》和《现代职业教育体系建设规划》的重点任务之一就是地方本科院校转 型发展 $[1,2]$ 。我国有近 1200 所高等院校, 地方高校近一半。在此政策公布两年多的时间, 各类地方院校已经开始积极准备转型发展的前期准备。作为五年制临床专业为主的地方医学 
院校, 由于专业特色, 对于此种应用转型尤为欢迎。将普通高等院校转型为技能型、应用型 的政策, 促使地方医学院校对各科课程开始了新一轮的教学改革热潮, 其中尤以基础类公共 课程改革力度最大, 引起的思考较多。本文将以医学院各类专业的公共基础课, 《医学物理学》 在这种政策作用下的改革为研究对象，研究开设改革课程的必要性问题。

\section{2. 地方医学院校《医学物理学》课程开设现状}

目前, 按照教育部规定, 在医学教育中必须包含对自然科学课程的学习。在我国地方医学院 校中, 《医学物理学》作为医学各专业学生必修公共基础课进行开设。《医学物理学》课程具 有两个基本功能: 第一, 为医学生提供系统的物理学知识, 训练医学生的逻辑思维分析以及 动手解决问题的能力。第二, 帮助医学生利用物理规律理解医学现象, 为今后学生进行交叉 学科研究提供相应地帮助。基于此功能, 《医学物理学》课程教学分为理论教学和实验教学。 以笔者所在的十堰地区医学院《医学物理学》课程开设为例, 学时针对专业的区别划分, 具 有一定的随意性, 缺乏科学依据。综合而言, 当下地方医学院校《医学物理学》课程设置, 缺乏科学性且定位模糊。因此, 在教育部出台高等院校转型的政策一旦提出, 再一次吹响了 对这类公共基础课程改革的号角, 怎么改? 笔者认为取决于怎样科学地解读转型政策。

\section{3. 高校转型应用型政策的不同解读}

国务院在 “十二五” 规划后期提出以培养学生就业能力为导向的教育改革布局。对此政策解 读众说纷纭。许多解读过于片面, 国家之所以决定高校转型, 主是因为我国没有对高等院校 进行分类管理, 考核方式偏向于研究 (学术), 导致部分地方院校办学特色不明确, 培养出来 的学生的定位也比较尴尬。再加上我国市场经济的成熟, 以市场为导向的教育政策的制定是 符合教育发展的科学性的 [2]。教育部重点鼓励地方院校整体进行培养模式的转变, 作为地方 院校的医学院如何解读高校转型政策, 将直接决定学校对于各类学科教学改革的方式。笔者 认为，对于地方医学院校而言，解读高校转型政策主要存在以下两个观点:

第一, 温和型改革: 即认为改革必须要循序渐进, 不能将医学培养模式直接等同于职业技能 培养模式，因为职业教育中的纯技能培养和教育部提出的应用型、技能型教育还是有区别的。 这种观点下的改革，不会激进的将现有的一些非专业课程直接删除。

第二, 激进型改革: 即认为教育提出转型就是希望从根本上扭转定位于服务地方经济, 重视 教学的地方院校培养模式模糊的现状, 是时候对一些非专业课程, 参考职业技术学院一样进 行相当彻底地删除，节省出大量时间用于培养医学生的临床技能等。

以上两种观点, 将直接影响地方医学院校对一些非专业课程, 例如《医用高等数学》, 《医学 物理学》等课程的教学改革, 下面将分别论述。

\section{4. 温和改革下《医学物理学》课程开设的必要性}

在转型政策温和解读观点下, 《医学物理学》课程的开设是有其必要性的。为了迎合地方医学 院校的转型, 弱化这类课程所占比例, 强化这类课程考核形式多样化 (减轻学生负担) 是必 须进行的。将课程学时进行消减是无法避免的, 只是, 在温和政策解读下的学时消减应该具 有更多地科学论据。一个学科的教学改革, 要全盘考虑学科的特点, 课程教学改革往往是牵 一发而动全身, 地方医学院校的主要管理者大多是医学背景, 因此并没有对自然科学课程进 行科学系统的论证。大学物理的内容大量应用了高等数学这门工具, 并且对血液流变学、生 物力学、量子化学、生物分子学等课程均有一定的关联。因此, 《医学物理学》课程开设的必 要性必然牵连了《医用高等数学》开设的必要性, 也必然牵扯到生物物理, 生物化学等后续 专业课程的一些知识的理解 [4]。

既然课程有必要开设, 那么如何制定不同专业的教学大纲, 分类分级教学就是一个复杂的科 学问题。现在地方医学院校对待一般公共课基础课程的改革措施就是消减学时 [6]。笔者认为, 
单纯的消减学时作为教学改革, 医学背景为主的教育管理者们应该科学思考以下几个问题:

(1) 高等教育中必修公共基础课程和专业课程的划分目的是什么? 如果如《医学物理学》等 必修公共基础课, 无法达到培养学生综合素质, 保留该课程的必要性质就是一个伪命题 [5]。

（2）如何保证一门学科能够实现其培养学生素养的功能? 这个问题依赖于课程的性质，是人 文素养? 还是自然科学素养? 是重视动手实践的训练? 还是重视人际交流的训练? 如果该课 程的必修性质科学地保留, 需要多少课时进行数量和质量的积累才能实现? 这个问题必须面 对 [6]。

（3）公共基础课程功能实现的检验方式, 是以完成了所修学分为标准, 还是应该长远地考察 学生后续学习专业知识中的理解应用等? 作为医学临床专业的学生, 在一年级如何理解高血 压症状的产生, 通过怎样的思考方式, 质疑各类教材中给出的人体血液在动静脉中的流速。 甚至, 如何理解近视眼、远视眼的矫正原理? 近代物理中何为 MRI、X 射线影像和核医学的危 害等 $[3]$ 。

因此, 一门公共基础课程开设的必要性, 不能简单理解成其存在性, 而不去科学地研究怎样 实现这门课程应该具有的功能性，本质上是错误理解了高校转型政策。

\section{5. 激进改革下《医学物理学》课程开设的不必要性}

在激进转型政策解读观点下，只要和医学专业技能不相关的，且没有政治政策性要求的基础 课, 都应该从必修课程剔除, 即《医学物理学》课程开设的不必要性成立。常见的做法就是 将类似于《医学物理学》、《医用高等数学》等课程的必修课性质改为非必修课。这种改革看 似有以下几个好处, 首先, 可以减轻医学院学生学习负担。其次, 加强学生自主性学习能力, 将自然科学类课程列入自主性学习, 有利于增强学生通过计算机网络自主学习 [7], 有利于开 展新形式教学改革。再次, 将自然科学类课程例如选修或者网络自主性学习, 可以尽可能地 节约公共课教师成本, 有利于学校财政。如果《医学物理学》自然科学课程开设的必要性不 成立，笔者认为需要回答以下几个问题:

(1) 医学是否只定位为经验学科? 对于复杂疾病的确诊, 治疗都需要大量医生进行会诊, 表 明医学并不只是一个经验学科, 那么这类会诊的推理能力, 物理学和数学能否起到了一定作 用，是值得思考的 $[8]$ 。

(2) 医学疾病的治疗、诊断及检测是否需要交叉学科支持?物理学是否能够提供某些支持? 如果医学不需要交叉学科支持, 那么医学怎么会有今天的成就? 这个问题理不顺, 激进删除 《医学物理学》等课程完全不成立。

（3）地方医学院校培养的学生进一步深造后, 学生自然科学思维方式的欠缺怎样弥补? 地方 医学院的定位是服务于当地经济, 但是以现在的招生规模以及各级地市级医院对学历的要求, 地方医学院校的学生考研率会越来越高, 如何面对在没有物理、数学等自然科学基础的科研 学习生活, 值得思考。

（4）教育部是否该修订公共基础课于医学院校? 修订基础课程的必修性质, 不是一所学校而 是一大批学校。以培养技能型学生为理由, 将这类课程直接删除, 对职业学校也许行得通, 对层次稍微高一点的高等教育是否适用, 值得思考 $[9,10]$ 。

一门课程开设的不必要性, 并不是简单地从学生学习时间, 学校教师成本等问题孤立思考的。 因此, 在笔者看来, 在高校转型政策下做出《医学物理学》课程开设不必要的结论, 是完全 不科学的。

\section{6. 结论}

本文站在一个非常特殊的时期，即在我国 “十二五” 收关年和 “十三五” 开端年，以教育部 发布支持高校转型技能型、实用型政策一年多时间, 在大量地方高校踊跃响应该政策的前提 下。以地方医学院校开设《医学物理学》类公共基础课程的必要性为切入点, 将可能存在的 
两种改革观点呈现于读者。在高校转型政策中, 公共基础课程的改革往往不受重视, 存在本 文所述的两种现象。综合而言, 高等教育中任何一科公共基础课程开设的 “必要性”, 不能是 只是将其进行名义上的保留, 即温和地使其具有 “存在性”, 而更不能是激进地以 “有用性” 直接将其定义为 “不必要性”。只有重视了一门课程的 “科学性” 才能真正理解教育部制定的 高校转型政策。最后, 谨以此文呼吁在即将到来的高校转型过程中, 特别是地方高校中专业 特色鲜明的医学院校, 对待必修的公共基础课程开设的必要性问题改革, 必须坚持科学调研, 科学论证, 才能真正科学地制定利校利生, 利国利民地改革政策, 也才从本质上符合了高等 教育的发展规律。

\section{参考文献}

[1] 董洪亮, 地方本科高校该转型了（教育视界- 聚焦部分本科院校转型（上））[N], 人民 日报，2014年5月8日，第18版：文教周刊

[2] 董洪亮, 地方本科院校怎样转型（教育视界-聚焦部分本科院校转型（下））[N], 人民 日报，2014年5月8日，第18版：文教周刊

[3] 王否, 医学物理学 (第8版) [M], 北京: 人民卫生出版社, 2013年版

[4] 颜红金, 医学物理学与医学课程相结合的研究 [J], 中国医学物理学杂志, 2014年第1期: 4718-4722.

[5] 王晓艳;纪绪财;王鹏程;谢晋东, 医学物理学特色专业建设的研究与思考[J], 中国医学物 理学杂志，2014年第4期: 5095-5100.

[6] 方立铭;李国柱;程桂平, 医学物理学课程教学改革的方向与创新 [J], 当代教育论坛, 2010 年第4期: 100-101.

[7] 喀蔚波, 医学物理学教育的现状与思考[J], 中国医学物理学杂志, 2002, 19(4): 198-200.

[8] 谈笑玲, 医学物理学教学改革与探索[J], 高教学刊, 2016(2):122-123.

[9] 李朝阳, 医学物理学实验教学内容重构与教学模式改革 $[\mathrm{J}]$, 数理医药学杂志, 2016(1) :12-14.

[10]穆爱霞, 医学物理学开设问题探究 [J], 大学物理实验, 2015, 28(5):132-135.

\section{References}

[1] Dong HL. Right time to transformation of local undergraduate colleges (Education vision focusing on the transformation of undergraduate colleges (I)) [N], People's daily, May 8, 2014, eighteenth edition: Education weekly.

[2] Dong HL. How to transformation of local undergraduate colleges (Education vision focusing on the transformation of undergraduate colleges (II)) [N], People's daily, May 8, 2014, eighteenth edition: Education weekly.

[3] Wang L. Medicine Physics (Eighth Edition) [M], Bei Jing: People's Health Publishing company, 2013.

[4] Yan HJ. Research on the combination of medical physics and medical curriculum [J], Chinese Journal of Medical Education, 2014, 31(1): 4718-4722. 
[5] Wang XY, Ji XC, Wang PC, Xie JD, Research and Consideration on the construction of characteristic specialty in medical physics[J], Chinese Journal of Medical Education, 2014, 31(4):5095-5100.

[6] Fang LM, Li GZ, Cheng GP, The direction and innovation of Reformation of Medical Physics Teaching[J], Contemporary Education Forum, 2010, 4: 100-101.

[7] Ka WB, Present situation and thinking of Medical Physics Education [J], Chinese Journal of Medical Education, 2002, 19(4): 198-200.

[8] Tan XiaoLing, Teaching reform and exploration of Medical Physics [J], Journal of Higher Education, 2016(2):122-123.

[9] LI Chao-yang, Reformulation the learning content of medical physics [J], Journal of Mathematical Medicine, 2016(1):12-14.

[10]MU Ai-xia, Exploration on the problems of Medical Physics [J], Physical Experiment of College, 2015, 28(5):132-135.

作者简介: 朱本超 (1982 年一), 男, 湖北武汉, 副教授, 主要从事医学物理学教学, 医学 影像物理学教学研究, E-mail: zbc@hbmu. edu. cn 